\title{
Efficacy of Atrazine based Post-Emergence Herbicide Mixtures on Weed Dynamics and Maize (Zea mays L.) Productivity in Sub-Humid Southern Plain of Rajasthan
}

\author{
G.R. Mali*, A. Verma, Bharat D. Malunjker, Roshan Choudhary, \\ S.L. Mundra and Mahendra Sharma
}

Rajasthan College of Agriculture, Maharana Pratap University of Agriculture \& Technology, Udaipur, Rajasthan 313001, India

*Corresponding author

\begin{tabular}{|c|c|}
\hline & A B S T RA C T \\
\hline Keywords & \multirow{4}{*}{$\begin{array}{l}\text { A field experiment was conducted on clay loam soil during kharif, } 2017 \text { at Instructional } \\
\text { Agronomy Farm, Rajasthan College of Agriculture, Udaipur to find out the effect of } \\
\text { herbicides and their tank mix application on weed dynamics, growth and yield of maize. } \\
\text { The post-emergence tank mix application of atrazine } 0.5 \mathrm{~kg} / \mathrm{ha}+\text { tembotrione } 0.125 \mathrm{~kg} / \mathrm{ha} \\
\text { at } 15 \mathrm{DAS} \text { reduced weed density, weed dry matter and improved weed control efficiency } \\
\text { of monocot, dicot and total weed at } 60 \text { DAS. The maximum number of cob/plant }(1.47) \text {, } \\
\text { cob weight } / \text { plant }(154.94 \mathrm{~g}) \text { and weight of grain } / \mathrm{cob}(119.17 \mathrm{~g}) \text {, number of grain row } / \mathrm{cob} \\
(15.33), \text { number of grain } / \mathrm{row}(29.67) \text {, test weight }(228.33 \mathrm{~g}) \text { and shelling percentage } \\
(76.94 \%) \text { were obtained by weed control through tank mix applicationn of atrazine } 0.5 \\
\mathrm{~kg} / \mathrm{ha}+\text { tembotrione } 0.125 \mathrm{~kg} / \mathrm{ha} \text { at } 15 \mathrm{DAS} \text {. Similarly, application of atrazine } 0.5 \mathrm{~kg} / \mathrm{ha}+ \\
\text { tembotrione } 0.125 \mathrm{~kg} / \mathrm{ha} \text { at } 15 \mathrm{DAS} \text { also resulted in significantly higher grain, stover and } \\
\text { biological yield compared to rest of treatments. Likewise, it was also superior most with } \\
\text { respect to all the yield attributes, yield and shelling\% as compared to the other treatment. }\end{array}$} \\
\hline $\begin{array}{l}\text { Weed dynamics, } \\
\text { Atrazine, } \\
\text { Tembotrione, Post- } \\
\text { emergence, Maize, } \\
\text { Yield }\end{array}$ & \\
\hline Article Info & \\
\hline & \\
\hline
\end{tabular}

Introduction

Maize (Zea mays L.) is the third most important cereal in the world; it is one of the most versatile emerging crops having wider adaptability under varied agro-climatic conditions. In India, maize occupies 9.25 million ha area with production and productivity of 23.67 million tonnes and 2.53 t /ha respectively. Maize is highly productive crop with diversified uses, mainly as food and feed for livestock. It is an important source of carbohydrate, protein, iron, vitamin $\mathrm{B}$, and minerals. Over $85 \%$ of its production in India is consumed as various processed foods like starch, corn syrup, popcorn, corn oil, corn flakes, roasted ears, biscuits, instant upma, instant kesari bhat, ready to eat maize puffs, chapaties. Apart from genetic and climatic factors the productivity of maize is very much affected by management issues. Among various factors of maize cultivation during rainy season weeds are considered to be of prime importance responsible for the low yield. The competition stress of weeds on crop for nutrients, water, light and space is 
responsible for poor yield of maize (Kumar et $a l .$, 2015). In Rajasthan, maize is grown mainly during rainy season and weather conditions of crop growing season are conducive to excessive weed infestation. The excessive occurrence of weeds limits the full expression of yield potential of this crop. Maize is infested by a wide range of weed flora; Commelina benghalensis, Echinochloa colona, Trianthema portulacastrum, Cyperus rotundus and Digera arvensis dominate during early stages of the crop growth whereas Dactyloctenium aegyptium and Physalis minima dominate toward the tasseling and maturity of the crop (Rao et al., 2009).

The most critical period for crop-weed competition is first six weeks after planting of crop which may reduce yield by $28-100 \%$ (Dass et al., 2012). During this period, weeding is essentially required physical and mechanical means are expensive and many times timely operations are not possible due to continuous rains in monsoon season (Chopra and Angiras, 2008). Thus, attention must be focused on weed control measures to maintain the competitive ability of the threatened crop by minimizing weed interference during the critical growth phase of the crop. Most of the available herbicides for maize are applied as pre-emergence and these not effectively control the weed flora emerged during critical period of crop-weed competition. Under the existing practice, application of single herbicide under diverse and mixed weed flora does not provide satisfactory weed control for the desired period. Moreover, continuous use of single herbicide is known to result in the evolution of herbicide-resistance in weed species and shift in weed flora (Thakur and Sharma, 1997, Malviya and Singh, 2007). Atrazine alone has been widely used as pre-emergence herbicides for controlling of the weeds in maize under monocultures (Barla et al., 2016).Considering these facts and paucity of research finding, the present study was planned with objective to evaluate the effect of atrazine based postemergence herbicide mixtures and their time of application on weed dynamics, growth and yield of maize.

\section{Materials and Methods}

The experiment was carried out at research farm of Raasthan college of Agriculture, Udaipur $\left(24^{\circ} 35^{\prime} \mathrm{N}\right.$ latitude and $73^{\circ} 42^{\prime} \mathrm{E}$ longitude.an altitude of 582.5 meter above mean sea level).The soil of experimental had low in nitrogen, medium in phosphorus, high in potassium and slightly alkaline and calcarious in nature.

The soil of the experimental field was clay loam in texture. The experiment was laid out in randomized block design with 13 treatment combination tested were as follows weedy check, atrazine $0.5 \mathrm{~kg} / \mathrm{ha}$ at $10 \mathrm{DAS}$, atrazine $0.5 \mathrm{~kg} / \mathrm{ha}$ at $15 \mathrm{DAS}$, atrazine $0.5 \mathrm{~kg} / \mathrm{ha}$ at 20 DAS, atrazine $0.5 \mathrm{~kg} / \mathrm{ha}+$ halosulfuron 0.09 $\mathrm{kg} / \mathrm{ha}$ at $10 \mathrm{DAS}$, atrazine $0.5 \mathrm{~kg} / \mathrm{ha}+$ halosulfuron $0.09 \mathrm{~kg} / \mathrm{ha}$ at $15 \mathrm{DAS}$, atrazine $0.5 \mathrm{~kg} / \mathrm{ha}+$ halosulfuron $0.09 \mathrm{~kg} / \mathrm{ha}$ at 20 DAS, atrazine $0.5 \mathrm{~kg} / \mathrm{ha}+$ tembotrione 0.125 $\mathrm{kg} / \mathrm{ha}$ at $10 \mathrm{DAS}$, atrazine $0.5 \mathrm{~kg} / \mathrm{ha}+$ tembotrione $0.125 \mathrm{~kg} / \mathrm{ha}$ at $15 \mathrm{DAS}$, atrazine $0.5 \mathrm{~kg} / \mathrm{ha}+$ tembotrione $0.125 \mathrm{~kg} / \mathrm{ha}$ at 20 DAS, atrazine $0.5 \mathrm{~kg} / \mathrm{ha}+$ topramezone 0.118 $\mathrm{kg} / \mathrm{ha}$ at $10 \mathrm{DAS}$, atrazine $0.5 \mathrm{~kg} / \mathrm{ha}+$ topramezone $0.118 \mathrm{~kg} / \mathrm{ha}$ at $15 \mathrm{DAS}$ and atrazine $0.5 \mathrm{~kg} / \mathrm{ha}$ + topramezone $0.118 \mathrm{~kg} / \mathrm{ha}$ at $20 \mathrm{DAS}$. The result were analyzed taking consideration of wed parameters such as as weed density, weed dry matter, weed control efficiency and plant parameter such as plant population, plant dry matter, plant height, number of cob/plant, cob weight/plant (g) and weight of grain/cob (g), number of grain row/cob, number of grain/row, test weight (g) and shelling percentage, grain yield, stover yield and biological yield. 
Weed control efficiency was calculated at 60 DAS using the following formula (Mani et al., 1968).

Grain samples were drawn from the produce after weighing the net yield of each plot.From these, 1000 grain were counted and weighing to record test weight $(\mathrm{g})$. Biological yield was calculated by summing up the weight of thoroughly, grain and stover and expressed in t/ha.

\section{Results and Discussion}

\section{Weed studies}

The data reflected that proportion of density of monocot to dicot weeds in weedy check plots at 60 DAS 1:0.36. Likewise the dry matter record in corresponding plots revealed that the monocot versus dicot weed proportion was 1:0.40 at 60 DAS. The magnitude of existence of monocot and dicot weeds indicated that the crop suffered stress from a mixed flora of weeds.

The results (Table 1) indicated that all herbicide treatments either alone or tank mix application caused marked reduction in weed density and dry matter of monocot, dicot and total weeds at 60 DAS.

However, the trend of effects of weed control treatments was not similar for monocot and dicot weeds. In respect of total weeds, postemergence application of atrazine $0.5 \mathrm{~kg} / \mathrm{ha}+$ tembotrione $0.125 \mathrm{~kg} / \mathrm{ha}$ at 15 DAS significantly reduced the weed density and total weed dry matter at 60 DAS followed by post-emergence application of atrazine 0.5 $\mathrm{kg} / \mathrm{ha}+$ topramezone $0.118 \mathrm{~kg} / \mathrm{ha}$ at $15 \mathrm{DAS}$ compared to other weed control treatments.

The results corroborate the findings of Stanzen et al., (2016) and Rana et al., (2017). Maximum weed control efficiency of total weeds at 60 DAS was observed with application of atrazine $0.5 \mathrm{~kg} / \mathrm{ha}+$ tembotrione $0.125 \mathrm{~kg} / \mathrm{ha}$ at 15 DAS $(97.49 \%)$ followed by atrazine $0.5 \mathrm{~kg} / \mathrm{ha}+$ topramezone $0.118 \mathrm{~kg} / \mathrm{ha}$ at $20 \mathrm{DAS}(96.91 \%)$.

\section{Crop studies}

Plant population of maize was not significantly affected by weed control treatments. It was clear from data in (Table $2)$. that combination of herbicide and herbicide alone have no significant influence on plant population at harvest.The postemergence application of atrazine $0.5 \mathrm{~kg} / \mathrm{ha}+$ tembotrione $0.125 \mathrm{~kg} / \mathrm{ha}$ at 15 DAS recorded the highest plant height at 60 DAS which was higher to rest of the treatments.

The per cent increase in plant height due to this treatment was $24.44 \%$, over weedy check. The maximum dry matter accumulation at 60 DAS was recorded under post-emergence application of atrazine $0.5 \mathrm{~kg} / \mathrm{ha}+$ tembotrione $0.125 \mathrm{~kg} / \mathrm{ha}$ at 15 DAS treatment with the corresponding per cent increase of $57.89 \%$ compared to weedy check. The maximum number of cob/plant (1.47), cob weight/plant (154.94 g), weight of grain/cob (119.17 g), number of grain row/cob (15.33), number of grain/row (29.67), test weight $(228.33 \mathrm{~g})$ and shelling percentage $(76.94 \%)$ were obtained by weed control through atrazine $0.5 \mathrm{~kg} / \mathrm{ha}+$ tembotrione $0.125 \mathrm{~kg} / \mathrm{ha}$ at 15 DAS. The result also has been reported by Singh et al., (2012), Rao et al., (2009), Nazreen and Subramanyam (2017).

The per cent increse in seed yield due to postemergence application of atrazine $0.5 \mathrm{~kg} / \mathrm{ha}+$ tembotrione $0.125 \mathrm{~kg} / \mathrm{ha}$ at $15 \mathrm{DAS}$ and postemergence application of atrazine $0.5 \mathrm{~kg} / \mathrm{ha}+$ tembotrione $0.125 \mathrm{~kg} / \mathrm{ha}$ at 15 DAS was 121.37 and 109.89 respectively, over weedy check. 
Table.1 Effect of treatments on weed density $\left(\mathrm{No} . / \mathrm{m}^{2}\right)$, weed dry matter $\left(\mathrm{g} / \mathrm{m}^{2}\right)$ and weed control efficiency $(\%)$ at 60 DAS in maize

\begin{tabular}{|c|c|c|c|c|c|c|c|c|c|}
\hline \multirow{3}{*}{ Treatments } & \multirow{2}{*}{\multicolumn{3}{|c|}{$\begin{array}{l}\text { Weed density* } \\
\text { 60 DAS }\end{array}$}} & \multirow{2}{*}{\multicolumn{3}{|c|}{$\begin{array}{c}\text { weed dry matter } \\
60 \text { DAS }\end{array}$}} & \multirow{2}{*}{\multicolumn{3}{|c|}{$\begin{array}{c}\text { weed control efficiency } \\
60 \text { DAS }\end{array}$}} \\
\hline & & & & & & & & & \\
\hline & $\begin{array}{l}\text { Monocot } \\
\text { weeds }\end{array}$ & $\begin{array}{l}\text { Dicot } \\
\text { weeds }\end{array}$ & $\begin{array}{c}\text { Total } \\
\text { weeds }\end{array}$ & $\begin{array}{l}\text { Monocot } \\
\text { weeds }\end{array}$ & $\begin{array}{l}\text { Dicot } \\
\text { weeds }\end{array}$ & $\begin{array}{l}\text { Total } \\
\text { weeds }\end{array}$ & $\begin{array}{l}\text { Monocot } \\
\text { weeds }\end{array}$ & $\begin{array}{l}\text { Dicot } \\
\text { weeds }\end{array}$ & $\begin{array}{l}\text { Total } \\
\text { weeds }\end{array}$ \\
\hline Weedy check & $\begin{array}{c}12.65 \\
(159.47)\end{array}$ & $\begin{array}{c}7.58 \\
(57.04)\end{array}$ & $\begin{array}{c}14.73 \\
(216.50)\end{array}$ & 104.93 & 42.46 & 147.40 & 0.00 & 0.00 & 0.00 \\
\hline Atrazine $0.5 \mathrm{~kg} \mathrm{ha}^{-1}$ at $10 \mathrm{DAS}$ & $\begin{array}{c}5.66 \\
(31.54)\end{array}$ & $\begin{array}{c}3.55 \\
(12.12)\end{array}$ & $\begin{array}{c}6.64 \\
(43.66)\end{array}$ & 19.78 & 8.98 & 28.76 & 81.13 & 78.70 & 80.45 \\
\hline Atrazine $0.5 \mathrm{~kg} \mathrm{ha}^{-1}$ at $15 \mathrm{DAS}$ & $\begin{array}{c}5.97 \\
(35.19)\end{array}$ & $\begin{array}{c}3.67 \\
(13.01)\end{array}$ & $\begin{array}{c}6.98 \\
(48.20)\end{array}$ & 22.24 & 9.63 & 31.87 & 78.80 & 77.30 & 78.38 \\
\hline Atrazine $0.5 \mathrm{~kg} \mathrm{ha}^{-1}$ at $20 \mathrm{DAS}$ & $\begin{array}{c}6.47 \\
(41.41)\end{array}$ & $\begin{array}{c}4.43 \\
(19.12)\end{array}$ & $\begin{array}{c}7.81 \\
(60.53)\end{array}$ & 25.76 & 14.12 & 39.88 & 75.45 & 66.70 & 72.93 \\
\hline Atrazine $0.5 \mathrm{~kg} \mathrm{ha}^{-1}+$ Halosulfuron $0.09 \mathrm{~kg} \mathrm{ha}^{-1}$ at $10 \mathrm{DAS}$ & $\begin{array}{c}5.15 \\
(26.03)\end{array}$ & $\begin{array}{c}3.05 \\
(8.84)\end{array}$ & $\begin{array}{c}5.95 \\
(34.86)\end{array}$ & 16.28 & 6.60 & 22.88 & 84.48 & 84.40 & 84.47 \\
\hline Atrazine $0.5 \mathrm{~kg} \mathrm{ha}^{-1}+$ Halosulfuron $0.09 \mathrm{~kg} \mathrm{ha}^{-1}$ at 15 DAS & $\begin{array}{c}5.11 \\
(25.64)\end{array}$ & $\begin{array}{c}3.48 \\
(11.59)\end{array}$ & $\begin{array}{c}6.14 \\
(37.23)\end{array}$ & 15.73 & 8.62 & 24.35 & 85.00 & 79.59 & 83.46 \\
\hline Atrazine $0.5 \mathrm{~kg} \mathrm{ha}^{-1}+$ Halosulfuron $0.09 \mathrm{~kg} \mathrm{ha}^{-1}$ at 20 DAS & $\begin{array}{c}6.25 \\
(38.55)\end{array}$ & $\begin{array}{c}3.85 \\
(14.36)\end{array}$ & $\begin{array}{c}7.31 \\
(52.91)\end{array}$ & 24.60 & 10.67 & 35.27 & 76.52 & 74.72 & 76.03 \\
\hline Atrazine $0.5 \mathrm{~kg} \mathrm{ha}^{-1}+$ Tembotrione $0.125 \mathrm{~kg} \mathrm{ha}^{-1}$ at 10 DAS & $\begin{array}{c}2.62 \\
(6.39)\end{array}$ & $\begin{array}{c}2.35 \\
(5.01)\end{array}$ & $\begin{array}{c}3.45 \\
(11.40)\end{array}$ & 4.00 & 3.71 & 7.71 & 96.19 & 91.26 & 94.78 \\
\hline Atrazine $0.5 \mathrm{~kg} \mathrm{ha}^{-1}+$ Tembotrione $0.125 \mathrm{~kg} \mathrm{ha}^{-1}$ at 15 DAS & $\begin{array}{c}1.96 \\
(3.34)\end{array}$ & $\begin{array}{c}1.57 \\
(1.97)\end{array}$ & $\begin{array}{c}2.41 \\
(5.31)\end{array}$ & 2.24 & 1.46 & 3.71 & 97.86 & 96.55 & 97.49 \\
\hline Atrazine $0.5 \mathrm{~kg} \mathrm{ha}^{-1}+$ Tembotrione $0.125 \mathrm{~kg} \mathrm{ha}^{-1}$ at 20 DAS & $\begin{array}{c}2.31 \\
(4.85)\end{array}$ & $\begin{array}{c}1.82 \\
(2.81)\end{array}$ & $\begin{array}{c}2.85 \\
(7.66)\end{array}$ & 3.18 & 2.09 & 5.27 & 96.97 & 95.10 & 96.43 \\
\hline Atrazine $0.5 \mathrm{~kg} \mathrm{ha}^{-1}+$ Topramezone $0.025 \mathrm{~kg} \mathrm{ha}^{-1}$ at 10 DAS & $\begin{array}{c}2.61 \\
(6.30)\end{array}$ & $\begin{array}{c}2.00 \\
(3.53)\end{array}$ & $\begin{array}{c}3.21 \\
(9.83)\end{array}$ & 4.10 & 2.62 & 6.73 & 96.09 & 93.80 & 95.43 \\
\hline Atrazine $0.5 \mathrm{~kg} \mathrm{ha}^{-1}+$ Topramezone $0.025 \mathrm{~kg} \mathrm{ha}^{-1}$ at $15 \mathrm{DAS}$ & $\begin{array}{c}2.10 \\
(3.91)\end{array}$ & $\begin{array}{c}1.71 \\
(2.44)\end{array}$ & $\begin{array}{c}2.62 \\
(6.35)\end{array}$ & 2.74 & 1.81 & 4.54 & 97.39 & 95.72 & 96.91 \\
\hline Atrazine $0.5 \mathrm{~kg} \mathrm{ha}^{-1}+$ Topramezone $0.025 \mathrm{~kg} \mathrm{ha}^{-1}$ at 20 DAS & $\begin{array}{c}2.76 \\
(7.10)\end{array}$ & $\begin{array}{c}2.72 \\
(6.88)\end{array}$ & $\begin{array}{c}3.81 \\
(13.98)\end{array}$ & 5.35 & 5.13 & 10.48 & 94.90 & 87.90 & 92.88 \\
\hline $\mathrm{SEm} \pm$ & 0.09 & 0.08 & 0.10 & 0.59 & 0.55 & 0.92 & \multirow[t]{2}{*}{ NA } & \multirow[t]{2}{*}{ NA } & \multirow[t]{2}{*}{ NA } \\
\hline $\operatorname{LSD}(\overline{\mathbf{P}}=\mathbf{0 . 0 5})$ & 0.26 & 0.23 & 0.29 & 1.71 & 1.59 & 2.70 & & & \\
\hline
\end{tabular}

$(* \sqrt{x+0.5}$ Transformed values and Data in parenthesis are original values); NA: Not analysed 
Table.2 Effect of herbicides on different yield attributes of maize

\begin{tabular}{|c|c|c|c|c|c|c|}
\hline Treatment & $\begin{array}{c}\text { Number } \\
\text { of } \\
\text { row/cob }\end{array}$ & $\begin{array}{c}\text { Number } \\
\text { of } \\
\text { grain/row }\end{array}$ & $\begin{array}{c}\text { Number } \\
\text { of } \\
\text { cob/plant }\end{array}$ & $\begin{array}{c}\text { Cob } \\
\text { weight }(g) / \text { plant }\end{array}$ & $\begin{array}{l}\text { Weight. of } \\
\text { grain }(g) / \text { cob }\end{array}$ & $\begin{array}{c}\text { Test } \\
\text { weight } \\
\text { (g) }\end{array}$ \\
\hline Weedy check & 12.00 & 22.00 & 1.07 & 117.77 & 68.00 & 210.00 \\
\hline Atrazine $0.5 \mathrm{~kg} \mathrm{ha}^{-1}$ at $10 \mathrm{DAS}$ & 13.33 & 25.67 & 1.12 & 147.62 & 106.08 & 213.33 \\
\hline Atrazine $0.5 \mathrm{~kg} \mathrm{ha}^{-1}$ at 15 DAS & 12.67 & 26.67 & 1.13 & 144.75 & 109.58 & 214.00 \\
\hline Atrazine $0.5 \mathrm{~kg} \mathrm{ha}^{-1}$ at $20 \mathrm{DAS}$ & 12.00 & 27.33 & 1.17 & 147.10 & 105.40 & 219.33 \\
\hline Atrazine $0.5 \mathrm{~kg} \mathrm{ha}^{-1}+$ Halosulfuron $0.09 \mathrm{~kg} \mathrm{ha}^{-1}$ at $10 \mathrm{DAS}$ & 12.67 & 25.33 & 1.20 & 135.27 & 88.40 & 212.33 \\
\hline Atrazine $0.5 \mathrm{~kg} \mathrm{ha}^{-1}+$ Halosulfuron $0.09 \mathrm{~kg} \mathrm{ha}^{-1}$ at $15 \mathrm{DAS}$ & 14.67 & 25.00 & 1.13 & 136.32 & 83.17 & 215.33 \\
\hline Atrazine $0.5 \mathrm{~kg} \mathrm{ha}^{-1}+$ Halosulfuron $0.09 \mathrm{~kg} \mathrm{ha}^{-1}$ at 20 DAS & 12.67 & 24.33 & 1.13 & 143.70 & 83.33 & 213.00 \\
\hline $\begin{array}{l}\text { Atrazine } 0.5 \mathrm{~kg} \mathrm{ha}^{-1}+\text { Tembotrione } 0.125 \mathrm{~kg} \mathrm{ha}^{-1} \text { at } 10 \\
\text { DAS }\end{array}$ & 13.33 & 27.67 & 1.27 & 151.74 & 114.23 & 219.33 \\
\hline $\begin{array}{l}\text { Atrazine } 0.5 \mathrm{~kg} \mathrm{ha}^{-1}+\text { Tembotrione } 0.125 \mathrm{~kg} \mathrm{ha}^{-1} \text { at } 15 \\
\text { DAS }\end{array}$ & 15.33 & 29.67 & 1.47 & 154.94 & 119.17 & 228.33 \\
\hline $\begin{array}{l}\text { Atrazine } 0.5 \mathrm{~kg} \mathrm{ha}^{-1}+\text { Tembotrione } 0.125 \mathrm{~kg} \mathrm{ha}^{-1} \text { at } 20 \\
\text { DAS }\end{array}$ & 14.67 & 27.67 & 1.37 & 152.33 & 105.55 & 224.33 \\
\hline $\begin{array}{l}\text { Atrazine } 0.5 \mathrm{~kg} \mathrm{ha}^{-1}+\text { Topramezone } 0.025 \mathrm{~kg} \mathrm{ha}^{-1} \text { at } 10 \\
\text { DAS }\end{array}$ & 14.00 & 27.33 & 1.33 & 147.10 & 104.17 & 218.33 \\
\hline $\begin{array}{l}\text { Atrazine } 0.5 \mathrm{~kg} \mathrm{ha}^{-1}+\text { Topramezone } 0.025 \mathrm{~kg} \mathrm{ha}^{-1} \text { at } 15 \\
\text { DAS }\end{array}$ & 14.67 & 28.67 & 1.40 & 152.85 & 113.53 & 226.67 \\
\hline $\begin{array}{l}\text { Atrazine } 0.5 \mathrm{~kg} \mathrm{ha}^{-1}+\text { Topramezone } 0.025 \mathrm{~kg} \mathrm{ha}^{-1} \text { at } 20 \\
\text { DAS }\end{array}$ & 12.67 & 27.67 & 1.33 & 147.10 & 112.67 & 223.33 \\
\hline $\mathrm{SEm} \pm$ & 0.67 & 0.53 & 0.07 & 1.66 & 2.11 & 3.05 \\
\hline $\operatorname{LSD}(\bar{P}=0.05)$ & 1.95 & 1.55 & 0.22 & 4.86 & 6.17 & 8.91 \\
\hline
\end{tabular}


Table.3 Effect of herbicides on plant population, plant height $(\mathrm{cm})$, plant dry matter $(\mathrm{g} / \mathrm{plant})$, grain yield (t/ha), stover yield $(\mathrm{t} / \mathrm{ha})$, biological yield ( $\mathrm{t} / \mathrm{ha})$ and shelling $\%$ in maize

\begin{tabular}{|c|c|c|c|c|c|c|c|}
\hline Treatment & $\begin{array}{c}\text { Plant } \\
\text { population } \\
\text { at harvest }\end{array}$ & $\begin{array}{c}\text { Plant } \\
\text { height } \\
\text { at } 60 \text { DAS }\end{array}$ & $\begin{array}{l}\text { Plant dry } \\
\text { matter } \\
\text { at } 60 \text { DAS }\end{array}$ & $\begin{array}{l}\text { Grain } \\
\text { yield }\end{array}$ & $\begin{array}{c}\text { Stover } \\
\text { yield }\end{array}$ & $\begin{array}{l}\text { Biological } \\
\text { yield }\end{array}$ & Shelling \% \\
\hline Weedy check & 56581 & 130.80 & 32.13 & 2.37 & 4.01 & 6.38 & 57.72 \\
\hline Atrazine $0.5 \mathrm{~kg} \mathrm{ha}^{-1}$ at $10 \mathrm{DAS}$ & 59636 & 133.93 & 36.67 & 3.82 & 5.62 & 9.44 & 71.90 \\
\hline Atrazine $0.5 \mathrm{~kg} \mathrm{ha}^{-1}$ at $15 \mathrm{DAS}$ & 60508 & 145.00 & 37.22 & 3.29 & 5.27 & 8.56 & 75.71 \\
\hline Atrazine $0.5 \mathrm{~kg} \mathrm{ha}^{-1}$ at $20 \mathrm{DAS}$ & 60726 & 145.33 & 34.73 & 3.58 & 4.89 & 8.46 & 71.68 \\
\hline Atrazine $0.5 \mathrm{~kg} \mathrm{ha}^{-1}+$ Halosulfuron $0.09 \mathrm{~kg} \mathrm{ha}^{-1}$ at $10 \mathrm{DAS}$ & 60290 & 139.07 & 29.53 & 3.23 & 4.72 & 7.94 & 65.35 \\
\hline Atrazine $0.5 \mathrm{~kg} \mathrm{ha}^{-1}+$ Halosulfuron $0.09 \mathrm{~kg} \mathrm{ha}^{-1}$ at 15 DAS & 58109 & 134.00 & 28.63 & 3.19 & 5.07 & 8.27 & 61.02 \\
\hline Atrazine $0.5 \mathrm{~kg} \mathrm{ha}^{-1}+$ Halosulfuron $0.09 \mathrm{~kg} \mathrm{ha}^{-1}$ at 20 DAS & 58327 & 140.67 & 29.00 & 3.59 & 5.38 & 8.97 & 58.01 \\
\hline Atrazine $0.5 \mathrm{~kg} \mathrm{ha}^{-1}+$ Tembotrione $0.125 \mathrm{~kg} \mathrm{ha}^{-1}$ at 10 DAS & 61550 & 150.67 & 45.07 & 4.83 & 7.02 & 11.85 & 75.32 \\
\hline Atrazine $0.5 \mathrm{~kg} \mathrm{ha}^{-1}+$ Tembotrione $0.125 \mathrm{~kg} \mathrm{ha}^{-1}$ at 15 DAS & 61550 & 168.00 & 50.73 & 5.24 & 7.03 & 12.27 & 76.94 \\
\hline Atrazine $0.5 \mathrm{~kg} \mathrm{ha}^{-1}+$ Tembotrione $0.125 \mathrm{~kg} \mathrm{ha}^{-1}$ at 20 DAS & 61356 & 154.47 & 44.50 & 4.96 & 6.88 & 11.85 & 69.30 \\
\hline Atrazine $0.5 \mathrm{~kg} \mathrm{ha}^{-1}+$ Topramezone $0.025 \mathrm{~kg} \mathrm{ha}^{-1}$ at 10 DAS & 60883 & 163.00 & 48.60 & 4.89 & 6.77 & 11.66 & 70.84 \\
\hline Atrazine $0.5 \mathrm{~kg} \mathrm{ha}^{-1}+$ Topramezone $0.025 \mathrm{~kg} \mathrm{ha}^{-1}$ at 15 DAS & 58423 & 166.73 & 49.73 & 4.64 & 6.95 & 11.58 & 74.26 \\
\hline Atrazine $0.5 \mathrm{~kg} \mathrm{ha}^{-1}+$ Topramezone $0.025 \mathrm{~kg} \mathrm{ha}^{-1}$ at 20 DAS & 57175 & 148.67 & 45.93 & 4.60 & 6.89 & 11.48 & 76.59 \\
\hline $\mathrm{SEm} \pm$ & 1473 & 4.49 & 2.74 & 232 & 250 & 344 & 1.59 \\
\hline LSD $(P=0.05)$ & NS & 13.12 & 8.01 & 679 & 729 & 1004 & 4.65 \\
\hline
\end{tabular}


The highest strover yield obtained with postemergence application of atrazine $0.5 \mathrm{~kg} / \mathrm{ha}+$ tembotrione $0.125 \mathrm{~kg} / \mathrm{ha}$ at 15 DAS $(7.03$ t/ha) which was closed to post-emergence application of atrazine $0.5 \mathrm{~kg} / \mathrm{ha}+$ tembotrione $0.125 \mathrm{~kg} / \mathrm{ha}$ at 10 DAS $(7.02$ t/ha) with per cent increase of 75.30 and 75.05, respectively over weedy check (4.01 t/ha). Amongst treatments, post-emergence application of atrazine $0.5 \mathrm{~kg} / \mathrm{ha}+$ tembotrione $0.125 \mathrm{~kg} / \mathrm{ha}$ at 15 DAS and postemergence application of atrazine $0.5 \mathrm{~kg} / \mathrm{ha}+$ tembotrione $0.125 \mathrm{~kg} / \mathrm{ha}$ at $20 \mathrm{DAS}$, postemergence application of atrazine $0.5 \mathrm{~kg} / \mathrm{ha}+$ tembotrione $0.125 \mathrm{~kg} / \mathrm{ha}$ at $10 \mathrm{DAS}$ gave better results being at par with each other. The per cent increase in biological yield due to these treatments was 92.42,85.81 and 85.81, respectively over weedy check. The postemergence application of atrazine $0.5 \mathrm{~kg} / \mathrm{ha}+$ tembotrione $0.125 \mathrm{~kg} / \mathrm{ha}$ at $15 \mathrm{DAS}$ was found statistically at par atrazine $0.5 \mathrm{~kg} / \mathrm{ha}+$ topramezone $0.118 \mathrm{~kg} / \mathrm{ha}$ at $15 \mathrm{DAS}$ with respect to shelling percentage (\%),test weight (g) and decreasing order of merit. The results corroborate the findings of Patel et al., (2006), Walia et al., (2007), Choudhary et al., (2013) and Rana et al., (2017).

\section{References}

Berla, S., Upasani, R. R., Puran, A. N. and Thakur, R. 2016. Weed management in maize (Zea mays). Indian Journal of Weed Science 48: 67-69.

Chopra, P. and Angiras, N. N. 2008. Effect of tillage and weed management on productivity and nutrient uptake of maize (Zea mays). Indian Journal of Agronomy 53: 66-69.

Choudhary, P., Nepalia, V. and Singh, D.2013. Effect of weed control and sulphur on productivity of quality protein maize (Zea mays), dynamics of associated weeds and relative nutrient uptake. Indian Journal of Agronomy 58:
$534-538$.

Dass, S., Kumar, A., Jat, S. L., Parihar, C. M., Singh, A. K., Chikkappa, G. K. and Jat, M. L. 2012. Maize holds potential for diversification and livelihood security. Indian Journal of Agronomy $57 \quad\left(3^{\text {rd }}\right.$ IAC Special Issue): 32-37.

Kumar, A., Kumar, J., Puniya, R., Mahajan, A., Sharma, N. and Stanzen, L. 2015. Weed management in maize-based cropping system. Indian Journal of Weed Science 47: 254-266.

Malviya, A. and Singh, B. 2007. Weed dynamics, productivity and economics of maize (Zea mays L.) as affected by integrated weed management under rainfed condition. Indian Journal of Agronomy 52: 321-324.

Mani, V.S., Gautam, K.C. and Chakraberty, T.K. 1968. Losses in crop yield in India due to weed growth. PANS 42: 142-158.

Nazreen, S., Subramanyam, D., Sunitha, N. and Umamahesh, V. 2017.Nutrient uptake of maize and its associated weeds as influenced by sequential application of herbicides. International Journal of Pure and Applied Bioscience 5: 496-500.

Patel, V. J., Upadhyay, P.N., Patel, J.B and Meisuriya, M.I. 2006. Effect of herbicide mixture on weeds in kharif maize (Zea mays L.) under middle Gujarat conditions. Indian Journal of Weed science 38: 54-57.

Rana, S. S., Badiyala, D., Sharma, N., Kumar, R., Pathania P. 2017. Impact of tembotrione on weed growth, yield and economics of maize (Zea mays L.) under mid hill conditions of Himachal Pradesh. Pesticide Research Journal 29: 27-34.

Rao, A. S., Ratnam, M. and Reddy, T. Y. 2009. Weed management in zero -till down maize. Indian Journal of Weed Science 41: 46-49.

Singh, V. P., Guru, S. K., Kumar, A., Akshita, 
B. and Tripathi, N. 2012. Bioefficacy of tembotrione against mixed weed complex in maize. Indian Journal of Weed Science 44: 1-5.

Stanzen, L., Kumar, A., Sharma, B.C., Puniya, R. and Sharma, A. 2016. Weed dynamics and productivity under different tillage and weed-management practices in maize (Zea mays)-wheat (Triticum aestivum) cropping sequence. Indian Journal of Agronomy 61: 449454.
Thakur, D. R. and Sharma, V. 1997. Integrated weed management in rainfed maize. Indian Journal of Weed Science 28: 207-208.

Umarani, N.K and Boi, P.G. 1982. Studies on weed control in bajra under condition. JAMU 72: 145-147.

Walia, U.S., Surjit Singh and Buta singh. 2007. Integrated control of hardy weeds in Maize (Zea mays L.). Indian. Journal of Weed Science 39: 17-20.

\section{How to cite this article:}

Mali, G.R., A. Verma, Bharat D. Malunjker, Roshan Choudhary, S.L. Mundra and Mahendra Sharma. 2019. Efficacy of Atrazine based Post-Emergence Herbicide Mixtures on Weed Dynamics and Maize (Zea mays L.) Productivity in Sub-Humid Southern Plain of Rajasthan. Int.J.Curr.Microbiol.App.Sci. 8(01): 2888-2895. doi: https://doi.org/10.20546/ijcmas.2019.801.306 